\title{
Da Vinci's A Bear Walking
}

An eerie likeness

between a bear's paw

and a human's hand.

We don't realize this

until the bear is skinned, splayed on a blue tarp,

cracked open for viewing. Hands hold the power

to grab, fend, and cuff;

protect the young, sustain.

Our hands can dart, miss

the mark, dispatch

the wild beast roaming

the dark woods, travelling

its enduring trails. 